\title{
Twenty-four hour noninvasive ventilation in Duchenne muscular dystrophy: A safe alternative to tracheostomy
}

\author{
Douglas A McKim MD FRCPC FCCP DABSM ${ }^{1,2}$, Nadia Griller BSc MD³, \\ Carole LeBlanc RRT ${ }^{4}$, Andrew Woolnough $\mathrm{MSc}^{4,5}$, Judy King PT $\mathrm{PhD}^{6}$
}

\begin{abstract}
DA McKim, N Griller, C LeBlanc, A Woolnough, J King. Twentyfour hour noninvasive ventilation in Duchenne muscular dystrophy: A safe alternative to tracheostomy. Can Respir J 2013;20(1):e5-e9.
\end{abstract}

BACKGROUND: Almost all patients with Duchenne muscular dystrophy (DMD) eventually develop respiratory failure. Once $24 \mathrm{~h}$ ventilation is required, either due to incomplete effectiveness of nocturnal noninvasive ventilation (NIV) or bulbar weakness, it is common practice to recommend invasive tracheostomy ventilation; however, noninvasive daytime mouthpiece ventilation (MPV) as an addition to nocturnal mask ventilation is also an alternative.

METHODS: The authors' experience with 12 DMD patients who used $24 \mathrm{~h}$ NIV with mask NIV at night and MPV during daytime hours is reported.

RESULTS: The mean ( \pm SD) age and vital capacity (VC) at initiation of nocturnal (only) NIV subjects were $17.8 \pm 3.5$ years and $0.90 \pm 0.40 \mathrm{~L}(21 \%$ predicted), respectively; and, at the time of MPV, $19.8 \pm 3.4$ years and $0.57 \mathrm{~L}$ (13.2\% predicted), respectively. In clinical practice, carbon dioxide $\left(\mathrm{CO}_{2}\right)$ levels were measured using different methods: arterial blood gas analysis, transcutaneous partial pressure of $\mathrm{CO}_{2}$ and, predominantly, by end-tidal $\mathrm{CO}_{2}$. While the results suggested improved $\mathrm{CO}_{2}$ levels, these were not frequently confirmed by arterial blood gas measurement. The mean survival on $24 \mathrm{~h}$ NIV has been 5.7 years (range 0.17 to 12 years). Of the 12 patients, two deaths occurred after 3.75 and four years, respectively, on MPV; the remaining patients continue on $24 \mathrm{~h}$ NIV (range two months to 12 years; mean 5.3 years; median 3.5 years).

CONCLUSIONS: Twenty-four hour NIV should be considered a safe alternative for patients with DMD because its use may obviate the need for tracheostomy in patients with chronic respiratory failure requiring more than nocturnal ventilation alone.

Key Words: Duchenne; Lung volume recruitment; Neuromuscular; Noninvasive ventilation; Ventilation

Imost all patients with Duchenne muscular dystrophy (DMD) Aeventually develop respiratory failure. This initially occurs at night $(1,2)$ and has been shown to improve with the use of nocturnal noninvasive ventilation (NIV) (3-5). In fact, the incidence of cardiac insufficiency as a cause of mortality may be more common as a consequence of effectively correcting respiratory failure (6-8). Unfortunately, even with appropriate NIV adjustments and vigilant clinical follow-up, hours of night-time ventilation alone may be insufficient and daytime hypercapnea develops (9). Often, the clinical course also includes impairment in swallowing and, as a result of this combination, the traditional approach is to introduce tracheostomy ventilation (10). There is some controversy regarding this approach, however, because noninvasive alternatives to tracheostomy have been demonstrated to be effective by a number of investigations involving this population $(4,6,9,11)$. A recent retrospective review compared the clinical complications of tracheostomized patients with DMD with those who were entirely NIV supported and found airway complications to be more frequent, with a greater

\section{Une ventilation non effractive continue en cas de dystrophie musculaire de Duchenne : une solution sécuritaire par rapport à la trachéotomie}

HISTORIQUE : Presque tous les patients ayant une dystrophie musculaire
de Duchenne (DMD) finissent par présenter une insuffisance respiratoire.
Lorsque la ventilation continue devient nécessaire, il est pratique courante de
recommander une trachéotomie effractive, en raison d'une efficacité incom-
plète de la ventilation non effractive (VNE) nocturne ou d'une atteinte bul-
baire. Cependant, il est également possible d'utiliser la ventilation non
effractive assistée par embout buccal (VEB) pendant le jour en plus de la
ventilation nocturne au masque. MÉTHODOLOGIE : Les auteurs présentent leur expérience auprès de 12 patients ayant la DMD qui ont utilisé la VNE continue au masque pendant la nuit et la VEB pendant le jour.

RÉSULTATS : Les sujets sous VNE nocturne (seulement) avaient un âge moyen ( \pm ÉT) et une capacité vitale $(\mathrm{CV})$ de $17,8 \pm 3,5$ ans et de $0,90 \pm 0,40 \mathrm{~L}$ ( $21 \%$ de la valeur prévue) à l'initiation, respectivement, et, au moment de la VEB, de 19,3 ans et de 0,57 L (13,2 \% de la valeur prévue), respectivement. En pratique clinique, on a mesuré leur taux de dioxyde de carbone $\left(\mathrm{CO}_{2}\right)$ au moyen de diverses méthodes : analyse des gaz artériels, pression partielle transcutanée du $\mathrm{CO}_{2}$ et, surtout, $\mathrm{CO}_{2}$ en fin d'expiration. Les résultats laissent supposer une amélioration des taux de $\mathrm{CO}_{2}$, qui n'était toutefois pas souvent confirmée par la mesure des gaz artériels. La survie moyenne sous VNE continue était de 5,7 ans (plage de 0,17 à 12 ans). Deux des 12 patients sous VEB sont décédés au bout de 3,75 et de quatre ans, respectivement. Les autres ont poursuivi la VNE continue (plage de deux mois à 12 ans; moyenne de 5,3 ans; médiane de 3,5 ans).

CONCLUSIONS : La VNE continue devrait être considérée comme une solution sécuritaire chez les patients ayant une DMD parce qu'elle peut permettre d'éviter une trachéotomie chez les patients ayant une insuffisance respiratoire chronique pour qui la ventilation nocturne seule ne suffit pas.

\footnotetext{
${ }^{1}$ Canadian Alternatives in Non-invasive Ventilation Program, Respiratory Rehabilitation Services and The Ottawa Hospital Sleep Centre; ${ }^{2}$ Department of Medicine, University of Ottawa, Ottawa; ${ }^{3}$ University of Toronto, Toronto; ${ }^{4}$ The Ottawa Hospital, Rehabilitation Centre; ${ }^{5}$ Institute for Rehabilitation Research and Development; ${ }^{6}$ Physiotherapy Program, School of Rehabilitation Sciences, Faculty of Health Sciences, University of Ottawa, Ottawa, Ontario Correspondence: Dr Douglas A McKim, Canadian Alternatives in Non-invasive Ventilation Respiratory Program, Rehabilitation Services and The Ottawa Hospital Sleep Centre, 505 Smyth Road, Room 1201, Ottawa, Ontario K1H 8M2. Telephone 613-737-7350 ext 75434, fax 613-736-9054,

e-mail dmckim@ottawahospital.on.ca
} 


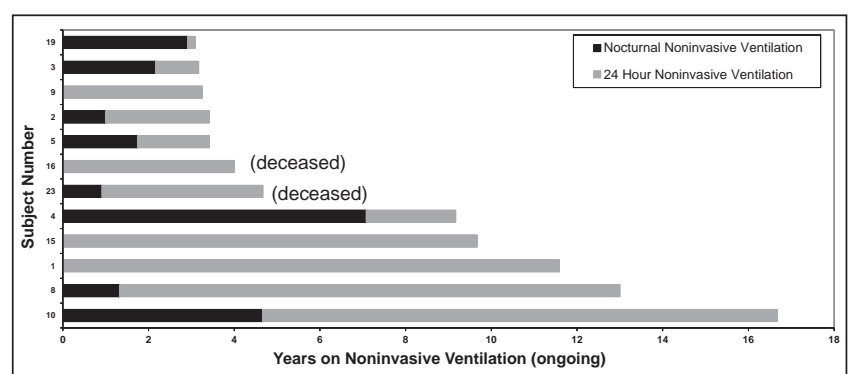

Figure 1) Survival to date of $24 \mathrm{~h}$ noninvasive mouthpiece ventilation in 12 ventilated patients. Two deaths (deceased), patients 16 and 23, are indicated. The remainder continue to survive on $24 \mathrm{~h}$ noninvasive ventilation

of our DMD patients was offered $24 \mathrm{~h}$ NIV/MPV. The purpose of the present study was to demonstrate the benefits to patients and the safety of using $24 \mathrm{~h}$ NIV.

\section{METHODS}

A retrospective chart analysis of consecutive patients with DMD who were referred to $\mathrm{TOH}$ over the past 15 years was conducted and identified patients who used $24 \mathrm{~h}$ NIV. The investigation was approved by TOH Ethics Review Board. Data were reviewed, where possible, from referring pediatric institutions as well as clinical assessments at the adult rehabilitation facility.

Of the 23 DMD patients (19 to 32 years of age) followed at the clinic, four have yet to require ventilatory support, seven continue with nocturnal NIV alone and 12 have used full-time NIV; nocturnal mask ventilation and daytime MPV (NIV/MPV), hereafter referred to as MPV. The average duration of follow-up on MPV is 4.6 years with a maximum of 13 years. Data collected from the 12 patients on continuous NIV/MPV formed the basis of the present study.

Objective evaluation at each visit included measures of $\mathrm{VC}$, forced expiratory volume in $1 \mathrm{~s}, 12 \mathrm{~s}$ maximum voluntary ventilation and maximum insufflation capacity (14) with lung volume recruitment (LVR, ie, 'breath stacking') (Profiler and CPSF/D, Medical Graphics Corporation, USA). Predicted values were those published by The National Health and Nutrition Examination Survey III (15). Additional measures included maximum inspiratory pressure (MIP) and maximum expiratory pressure (MEP) and cough peak flow (CPF). MIPs and MEPs were measured using a Micro RPM (Micro Medical Ltd, United Kingdom) or a manometer (Boehringer Laboratories, USA), and predicted values were those reported by Black and Hyatt (16). Cough flows were measured by instructing the patient to cough forcefully into a peak flow meter (Mini-Wright, Clement Clarke International Ltd, United Kingdom) and, whenever possible, under three different conditions: spontaneous (no volume or pressure assistance- $\left.\mathrm{CPF}_{\mathrm{SP}}\right)$, with the addition of $\mathrm{LVR}\left(\mathrm{CPF}_{\mathrm{LVR}}\right)$ and with the combination of LVR and a manually assisted cough (MAC), (CPF LVR-MAC when indicated for $\mathrm{CPF}_{\mathrm{LVR}}$ below $270 \mathrm{~L} / \mathrm{min}$ (11). The patient is required to hold their breath once the additional volume has been added and until the peak flow meter or pulmonary function mouthpiece has been applied and then release the total volume for the measurement of flow or volume. Oxyhemoglobin saturation was measured using pulse oximetry (Nellcor N-20P; Covidien, USA) and awake arterial carbon dioxide $\left(\mathrm{CO}_{2}\right)$ tension was measured using an end-tidal $\mathrm{CO}_{2}$ monitor (OxiMax NPB-75, USA), a transcutaneous $\mathrm{CO}_{2}$ monitor (Linde MicroGas 7650, Linde, Switzerland) or arterial blood gases when the end-tidal $\mathrm{CO}_{2}$ monitor was not available. Adequate ventilatory support was assessed using a combination of clinical assessment, normal overnight oximetry, serial daytime measures of $\mathrm{CO}_{2}$ and downloaded data from the bilevel devices. Because $\mathrm{CO}_{2}$ results were obtained by different methods, they are not reported as specific outcomes or analyzed for statistical differences. All $\mathrm{CO}_{2}$ measurements, including arterial blood gases, were performed when the patients were using no ventilatory support.
Nocturnal NIV was provided with bilevel devices (BiPAP S/T and Synchrony; Respironics, USA) or volume-cycled ventilators (LP6 Plus and LP10; Covidien, USA and LTV 1000; Pulmonetics, USA). Back-up rates were provided during bilevel ventilation using the pressurecontrol mode with a set inspiratory and rise time. Daytime MPV was provided with an LP6/10 or LTV, each with a $15 \mathrm{~mm}$ angled mouthpiece (Malincrodt-Puritan-Bennett, USA) or a length of tubing (Tygon, Saint Gobain Performance Products, USA) with tidal volumes $(\mathrm{Vt})$ and rate set to patient comfort.

All patients initiating NIV/MPV were treated in the outpatient clinic without hospital admission. No patient was excluded from MPV as a result of bulbar impairment. No DMD patient, yet encountered, has been unable to retain the mouthpiece or Tygon tubing as a result of weakness. Each patient and their caregivers were taught to perform airway clearance techniques including LVR, MAC techniques and mechanical insufflation-exsufflation (MI-E). Patients were encouraged to integrate these airway clearance techniques into their daily routine and use their chair-mounted ventilators to perform regular LVR using the mouthpiece.

\section{RESULTS}

The mean nocturnal bilevel inspiratory positive airway pressure (IPAP)/expiratory positive airway pressure (EPAP) pressures were $17 / 6 \mathrm{cmH}_{2} \mathrm{O}$ (EPAP range $4 \mathrm{cmH}_{2} \mathrm{O}$ to $13 \mathrm{cmH}_{2} \mathrm{O}$, IPAP range $12 \mathrm{cmH}_{2} \mathrm{O}$ to $24 \mathrm{cmH}_{2} \mathrm{O}$ ) and the mean volume cycled ventilator volumes were $900 \mathrm{~mL}$ with a respiratory rate of $14 / \mathrm{min}$. Data on specific back-up rates were available for bilevel devices. During daytime hours, patients on diurnal support used MPV $(14,17-19)$ in the assist/control mode, $\mathrm{Vt} 800 \mathrm{~mL}$ to $1100 \mathrm{~mL}$, respiratory rate $1 / \mathrm{min}$ (LTV) or 6/min (LP-6/10) with an inspiratory time of $1.2 \mathrm{~s}$ to $1.5 \mathrm{~s}$. Patients easily triggered the ventilator with mouth pressure to obtain as frequent a $\mathrm{Vt}$ as desired. One patient required a temporary tracheostomy for anaphylaxis and upper airway obstruction but was later returned to MPV and his stoma was closed. Another patient was extubated and returned to $24 \mathrm{~h} \mathrm{NIV}$, including MPV in the intensive care unit, on day 5 after a laparotomy for gastric perforation and abdominal sepsis. None of the 12 patients has required a permanent tracheostomy.

Over the course of 85 patient-years of full-time NIV/MPV, three respiratory-related hospitalizations and two deaths occurred (Figure 1). One patient (patient 16) died while on $24 \mathrm{~h}$ NIV at 26 years of age due to a flu-like illness, dehydration and refusal to go to hospital. He was known to have moderate left ventricular impairment. Another patient (patient 23) died while on $24 \mathrm{~h} \mathrm{NIV}$ at 20 years of age. He had extensive bilateral pneumonia and initially declined hospital admission or intubation. A remarkable observation in this patient was that in spite of severe hypoxemic respiratory failure, on mask ventilation alone, his $\mathrm{PaCO}_{2}$ remained normal, indicating normal alveolar ventilation. The third patient did not experience a complication as a result of MPV. During this evaluation, there were no adverse consequences of MPV such as accidental disconnections.

Years of MPV use by 12 patients is illustrated in Figure 1. The longest-living patient has used nocturnal NIV alone for 4.6 years with the addition of MPV for an additional 12 years. This patient continues to be successfully ventilated. Mean survival on MPV is 5.7 years (range 0.17 to 12 years).

All 12 patients began nocturnal NIV at a mean ( \pm SD) of $17.8 \pm 3.5$ years, with a VC of $0.90 \pm 0.40 \mathrm{~L}$ ( $21 \%$ predicted); MPV was added when patients presented with symptoms of diurnal respiratory failure including dyspnea or tachypnea or for increasing daytime $\mathrm{CO}_{2}$. The mean VC of these 12 patients was $0.57 \mathrm{~L}$ (13.2\% predicted) and the mean age was $19.8 \pm 3.4$ years. During the period of NIV use followed by daytime MPV, a gradual loss of pulmonary function was observed (Figure 2), including a reduction in forced vital capacity from $0.9 \mathrm{~L}$ to $0.5 \mathrm{~L}(\mathrm{P}<0.02)$ and a reduction in MIPs: -29.9 to -17.7 and MEPs $28.5 \mathrm{cmH}_{2} \mathrm{O}$ to $19 \mathrm{cmH}_{2} \mathrm{O}$ (each $\mathrm{P}<0.002$ ). To date, all but one patient has retained a measurable spontaneous $\mathrm{VC}$ in the range of $5 \%$ to $10 \%$ predicted. 


\section{DISCUSSION}

Twenty-four hour NIV should be considered a safe alternative for patients with DMD because its use may obviate the need for tracheostomy in patients with chronic respiratory failure requiring more than nocturnal ventilation alone.

The present study illustrates that, when provided with an informed choice of tracheostomy or NIV, all patients with DMD and families chose $24 \mathrm{~h}$ NIV including MPV. This investigation also demonstrated that temporary invasive ventilation can be provided and successfully converted to full-time NIV including MPV. Furthermore, these results illustrate that, in the face of an increasing requirement for ventilatory support, the addition of daytime MPV results in continued survival in spite of further decline in VC and MIP. In fact, there was a stable or increasing average $\mathrm{VC}$ in the 12 patients on MPV, possibly due to improvements respiratory mechanics in patients introduced to LVR at the same time or able to perform more regular, autonomous LVR with the mouthpiece.

Twenty-four hour NIV is not a new approach to respiratory failure $(4,20)$. Neuromuscular diseases, such as polio, have been managed in this fashion for decades, originally with negative-pressure body ventilators and, more recently, with positive pressure mask ventilation. During the polio era, it was well recognized that patients otherwise supported by iron lungs could be liberated from their tanks with positive pressure mouthpieces (21). Bach et al (4) and others have reported a large number of patients with neuromuscular diseases managed long beyond the point of respiratory failure with $24 \mathrm{~h}$ NIV. Even patients previously ventilated $24 \mathrm{~h}$ per day via a tracheostomy have been converted entirely to NIV/MPV (22).

The success of long-term NIV is highly dependent on the regular performance of effective techniques of airway clearance $(6,9,11,17,23)$. Some studies indicating a less than favourable outcome from NIV have been criticized for not including airway clearance strategies (24). It has been suggested that all investigations of NIV in patients with NMD should include details of techniques to improve cough and airway clearance (23); many recent outcome studies of NIV include such treatments $(6,9,25)$. We recently demonstrated an improvement in the rate of decline of forced vital capacity in DMD as a result of regular LVR (26). Each of our patients was trained in LVR using a hand-held resuscitation bag, and their performance was visually demonstrated to them with numerical values and illustrations of their pulmonary function. Provision of MPV enabled adequate daytime ventilation as well as the regular, autonomous performance of LVR desired by the patient. These principles were reinforced at each clinical visit and by access to an educational website (26). Seventy per cent of our patients had an MI-E device (CoughAssist, Philips Healthcare, USA) device in their homes and the remainder were provided education in the use of the MI-E and a prescription for its use when necessary (27-29). The consistent use of LVR and the MI-E may have contributed to the longterm success of NIV and the small number of hospital admissions. While tracheostomy ventilation may be necessary in patients with DMD with severe impairment of oropharyngeal musculature or with cognitive impairment, most patients are quite capable of using MPV. Many centres would advocate the use of tracheostomy ventilation once levels of daytime $\mathrm{CO}_{2}$ begin to rise while on nocturnal NIV or when the daily requirement for mask ventilation reaches some arbitrary number of hours (eg, $16 \mathrm{~h}$ ). This is likely due to unfamiliarity with $24 \mathrm{~h}$ NIV and in spite of current recommendations. The American Thoracic Society consensus statement and the Canadian Thoracic Society home ventilation guidelines suggest considering daytime MPV for patients with DMD $(11,30)$. Tracheostomy ventilation offers an apparent degree of security for the patient; however, it is associated with a number of serious complications that may well offset any advantage provided by an artificial airway (4). Invasive ventilation has also been associated with a higher frequency of institutional care and this factor alone may be sufficient for a patient to decline a tracheostomy even if this results in death. In our experience, all of our patients with DMD have benefited from $24 \mathrm{~h} \mathrm{NIV/MPV} \mathrm{as} \mathrm{a} \mathrm{means} \mathrm{to}$

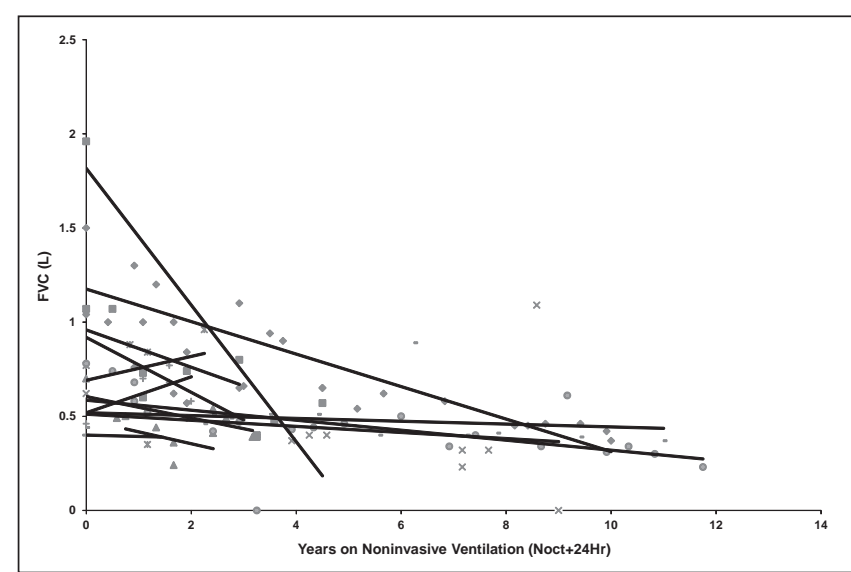

Figure 2) Decline of forced vital capacity (FVC) over years of nocturnal noninvasive ventilation followed by daytime mouthpiece ventilation. Noct Nocturnal

ensure adequate ventilation while avoiding a tracheostomy. Tracheostomy should be considered "when contraindications or patient aversion to noninvasive ventilation are present, or when noninvasive ventilation is not feasible due to severe bulbar weakness or dysfunction" (11). MPV offers the patient and family effective management without surgical risk, long-term complexity and the additional nursing care required with tracheostomy management. Tracheostomy ventilation can still be offered if and when necessary. One patient was referred from another centre while using nocturnal NIV with an arterial $\mathrm{CO}_{2}$ pressure of $75 \mathrm{mmHg}$ and detailed recommendations for urgent tracheostomy surgery, gastrostomy placement, inpatient ventilation training and long-term home mechanical ventilation. Instead, this young man was seen in three outpatient visits with adjustments to his bilevel device, introduction of LVR and daytime MPV. Gas exchange was normalized, quality of life improved and no tracheostomy has been required, even seven years later.

Clear physiological advantages of MPV have also been demonstrated. Toussaint et al (9) recently reported the presence of a fatiguing pattern of breathing using a noninvasive estimation of the tensiontime index in DMD patients who used night-time NIV but reported breathlessness in the late afternoon (31). Both the tension-time index and respiratory endurance against an inspiratory load were improved as a result of the introduction of only $2 \mathrm{~h}$ of MPV in the afternoon. Thus, it appears that the onset of daytime muscle fatigue is associated with symptoms of dyspnea and can be corrected with daytime MPV. It should be noted that in contrast to the Belgian studies $(9,31)$, all patients in our care were managed entirely as outpatients with the attendant cost savings. Although it is common practice to admit such patients to hospital for a short period of accommodation or to perform sleep studies, investigators have demonstrated that the outcomes of outpatient-initiated NIV are similar to those achieved as inpatients without the added complexity and cost of inpatient care $(32,33)$.

There may be concerns about aspiration risk with progressive bulbar weakness. These data represent all of our patients regardless of bulbar status. Patients with significant bulbar weakness were excluded from the population reported by Toussaint et al (9).

Both in our clinical experience and that of others, dysphagia may improve significantly with the introduction of MPV (9). The mechanism for the improvement in dysphagia is uncertain. It is possible that the reduction in work of breathing once MPV is initiated is associated with global improvements in muscle function. It is possible that there is also improvement in oropharyngeal muscle strength or endurance. It is also possible that the reduction in dyspnea that occurs during MPV allows for more comfortable swallowing and less anxiety during meals. It is recognized that adequate subglottic pressure is necessary to enable safe swallowing, a cause for difficulty in swallowing with 
a tracheostomy in place (34). It is possible that with MPV and the consequent ability to breathstack and increase lung volumes and, presumably, subglottic pressure, that the swallowing mechanisms may improve and support better nutrition (35).

The present study had important limitations. All of the data were reviewed retrospectively and were collected during clinical care in a busy outpatient environment in which conditions could not be controlled. As a result, there are many missing data. Some information was also obtained from referring centres and quality could not be confirmed. Although a prospective approach would also improve the evidence, a randomized study could not be performed due to obvious ethical issues. The measurements of $\mathrm{CO}_{2}$ were obtained using three different methods and this strategy respects the patients' desire not to undergo frequent arterial punctures. When combined with clinical evaluation, overnight oximetry and downloaded information from noninvasive devices, this is perfectly adequate for clinical care but was not used for statistical analysis.

All of the patients in the present study were cared for at home, not in an institution. While this is clearly associated with a better quality of life for the patient and a lower cost to the health care system, there is an enormous caregiving responsibility assumed by the family. As such, they each had dedicated family members who were ever-present to apply head gear, guarantee access to MPV and to provide effective, sometimes constant, airway clearance during respiratory tract infections or as a result of minor aspiration.

\section{REFERENCES}

1. Khan Y, Heckmatt JZ. Obstructive apnoeas in Duchenne muscular dystrophy. Thorax 1994;49:157-61.

2. Ragette R, Mellies U, Schwake C, Voit T, Teschler H. Patterns and predictors of sleep disordered breathing in primary myopathies. Thorax 2002;57:724-8.

3. Simonds AK, Muntoni F, Heather S, Fielding S. Impact of nasal ventilation on survival in hypercapnic Duchenne muscular dystrophy. Thorax 1998;53:949-52.

4. Bach JR, Alba AS, Saporito LR. Intermittent positive pressure ventilation via the mouth as an alternative to tracheotomy for 257 ventilator users. Chest 1993;103:174-82.

5. Ward S, Chatwin M, Heather S, Simonds AK. Randomised controlled trial of non-invasive ventilation (NIV) for nocturnal hypoventilation in neuromuscular and chest wall disease patients with daytime normocapnia. Thorax 2005;60:1019-24.

6. Gomez-Merino E, Bach JR. Duchenne muscular dystrophy: Prolongation of life by noninvasive respiratory muscle aids. Am J Phys Med Rehabil 2002;81:411-5.

7. Bach JR, Rajaraman R, Ballanger F, et al. Neuromuscular ventilatory insufficiency: The effect of home mechanical ventilator use vs. oxygen therapy on pneumonia and hospitalization rates. Am J Phys Med Rehabil 1998;77:8-19.

8. Rideau Y, Gatin G, Bach J, Gines G. Prolongation of life in Duchenne muscular dystrophy. Acta Neurol 1983;5:118-24.

9. Toussaint M, Steens M, Wasteels G, Soudon P. Diurnal ventilation via mouthpiece: Survival in end-stage Duchenne patients. Eur Respir J 2006;28:549-55.

10. Lofaso F, Orlikowski D, Raphael JC. Ventilatory assistance in patients with Duchenne muscular dystrophy. Eur Respir J 2006;28:468-9.

11. Finder JD, Birnkrant D, Carl J, et al. American Thoracic Society. Respiratory care of the patient with Duchenne muscular dystrophy: ATS consensus statement. Am J Respir Care Med 2004;170:456-65.

12. Soudon P, Steens M, Toussaint M. A comparison of invasive versus noninvasive full-time mechanical ventilation in Duchenne muscular dystrophy. Chr Resp Dis 2008;5:87-93.

13. Brooks D, King A, Tonack M, Simson H, Gould M, Goldstein R. User perspectives on issues that influence the quality of daily life of ventilator-assisted individuals with neuromuscular disorders. Can Respir J 2004;11:547-54.

14. Kang SW, Bach JR. Maximum insufflation capacity. Chest 2000;118:61-5.

15. Hankinson JL, Odencrantz JR, Fedan KB. Spirometric reference values from a sample of the general U.S. population. Am J Respir Crit Care Med 1999;159:179-87.
Given the relatively short follow-up period, it is possible that a number of our DMD patients will eventually require and will choose tracheostomy ventilation due to the development of severe bulbar weakness and aspiration risk. However, to date, patients who have been advised to undergo tracheostomies in other centres have been very successful and, in 85 patient-years, full time NIV/MPV has ensured survival in the face of declining respiratory function beyond the point of respiratory failure in these patients with DMD. Strong consideration should be given to $24 \mathrm{~h}$ NIV/MPV for all patients with DMD rather than routine recommendation for tracheostomy.

ACKNOWLEDGEMENTS: Douglas McKim contributed to this article by providing all of the medical care for these individuals, conceiving of the review and overseeing the data collection and preparing the manuscript. Nadia Griller and Andrew Woolnough extracted the data, provided data analysis and prepared all of the illustrations. Carole LeBlanc provided much of the respiratory therapy for each of the patients, contributed significantly to the conception of the review and the content of the manuscript. Judy King helped with the preparing of the manuscript. The authors also acknowledge the assistance of Christie O'Connell and Carolyn Cook of the the Institute for Rehabilitation Research Development, who provided assistance with the illustrations and some statistical analysis.

DISCLOSURES: The authors have no financial disclosures or conflicts of interest to declare.

16. Black LF, Hyatt RE. Maximal respiratory pressures: Normal values and relationship to age and sex. Am Rev Respir Dis 1969;99:696-702.

17. Bach JR. Prevention of morbidity and mortality with the use of physical medicine aids. In: Bach JR, ed. Pulmonary Rehabilitation: The Obstructive and Paralytic Conditions. Philadelphia: Hanley \& Belfus, 1996:303-29.

18. Boitano LJ, Benditt JO. An evaluation of home ventilators that support open circuit mouthpiece ventilation. Respir Care 2005;50:1457-61.

19. McKim DA, LeBlanc C. Maintaining an "oral tradition": Specific requirements for mouthpiece ventilation instead of tracheostomy for neuromuscular disease. Respir Care 2006;51:297-8.

20. Baydur A, Layne E, Aral H, et al. Long term non-invasive ventilation in the community for patients with musculoskeletal disorders: 46 year experience and review. Thorax 2000;55;4-11.

21. Affeldt JE. Roundtable conference on poliomyelitis equipment. 1953. National Foundation for Infantile Paralysis-March of Dimes. White Plains, New York.

22. Bach JR. A comparison of long-term ventilatory support alternatives from the perspective of the patient and care giver. Chest 1993;104:1702-6.

23. Servera E, Sancho J, Zafra MJ, Marin J. Secretion management must be considered when reporting success or failure of noninvasive ventilation. Chest 2003;123:1773-4.

24. Raphael JC, Chevret S, Chastang C. Randomised trial of preventive nasal ventilation in Duchenne muscular dystrophy. Lancet 1994;343:1600-4.

25. Bourke SC, Tomlinson M, Williams TL, Bullock RE, Shaw PJ, Gibson GJ. Effects of non-invasive ventilation on survival and quality of life in patients with amyotrophic lateral sclerosis: A randomised controlled trial. Lancet Neurol 2006;5:140-7.

26. McKim DA, Katz SL, LeBlanc C, et al. Lung volume recruitment slows lung function decline in Duchenne muscular dystrophy. Arch Phys Med Rehabil 2012;93:1117-22.

27. McKim D, LeBlanc C, Walker K, et al. Respiratory care protocols for spinal cord injuries and neuromuscular diseases. 2002. Institute for Rehabilitation Research and Development. <www.irrd.ca/ education> (Accessed June 9, 2012).

28. Gomez-Merino E, Sancho J, MarinJ, et al. Mechanical insufflationexsufflation: Pressure, volume, and flow relationships and the adequacy of the manufacturer's guidelines. Am J Phys Med Rehabil 2002;81:579-83.

29. Sancho J, Servera E, Marin J, Vergara P, Belda FJ, Bach JR. Effect of lung mechanics on mechanically assisted flows and volumes. Phys Med Rehabil 2004;83:698-703. 
30. McKim DA, Road J, Avendano M, et al. Canadian Thoracic Society clinical practice guidelines: Home mechanical ventilation. Can Respir J 2011;18:197-215.

31. Toussaint M, Soudon P, Kinnear W. Effect of non-invasive ventilation on respiratory muscle loading and endurance in patients with Duchenne muscular dystrophy. Thorax 2008;63;430-4.

32. Chatwin M, Nichol AH, Morrell MJ, Polkey MI, Simonds AK. Randomised trial of inpatient versus outpatient initiation of home mechanical ventilation in patients with nocturnal hypoventilation. Respir Med 2008;102:1528-35.
33. Lujan M, Moreno A, Veigas C, Monton C, Pomares X, Domingo C. Non-invasive mechanical ventilation: Effectiveness and efficiency of an outpatient initiation protocol compared with the standard in-hospital model. Respir Med 2007;101:1177-82.

34. Gross RD, Mahlmann J, Grayhack JP. Physiologic effects of open and closed tracheostomy tubes on the pharyngeal swallow. Ann Otol Rhinol Laryngol 2003;112:143-52.

35. Gross RD, Atwood CW, Grayhack JP. Lung volume effects on pharyngeal swallowing physiology. J Appl Physiol 2003;95:2211-7. 


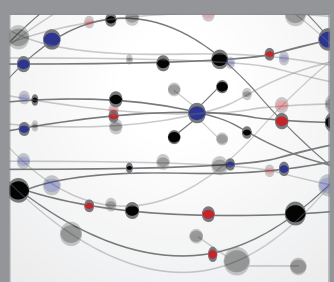

The Scientific World Journal
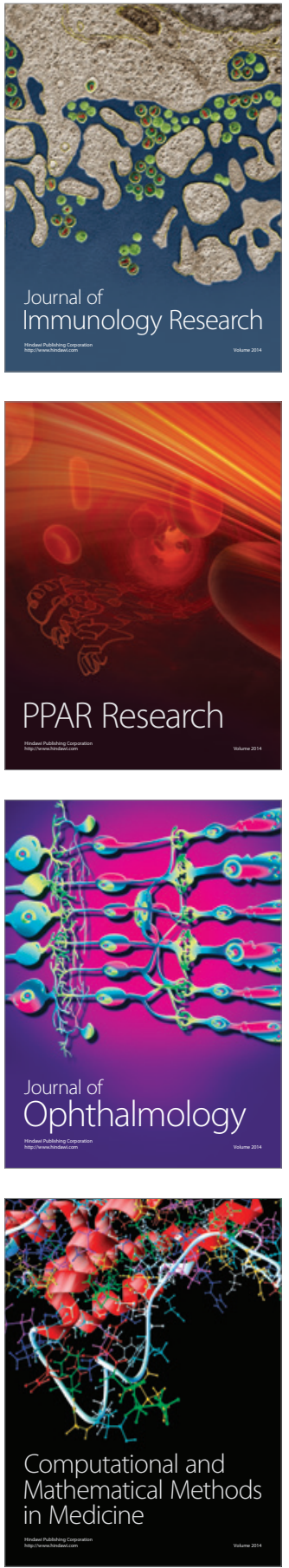

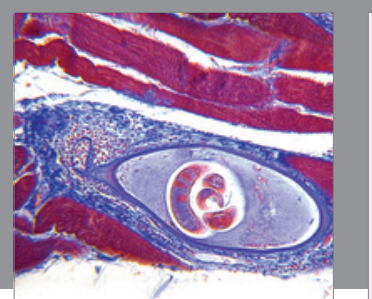

Gastroenterology Research and Practice

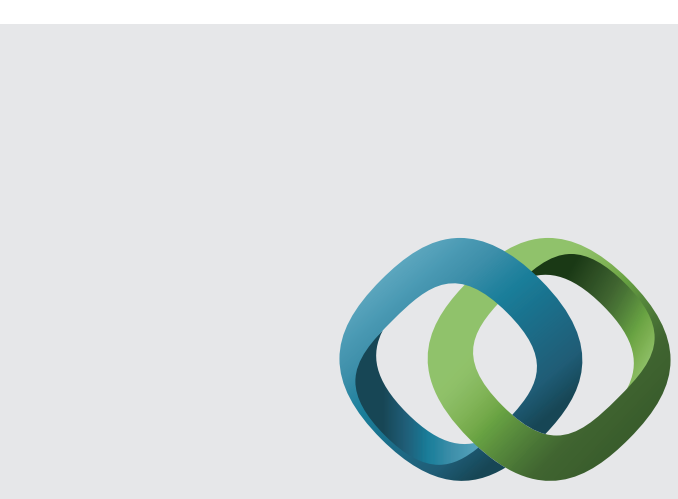

\section{Hindawi}

Submit your manuscripts at

http://www.hindawi.com
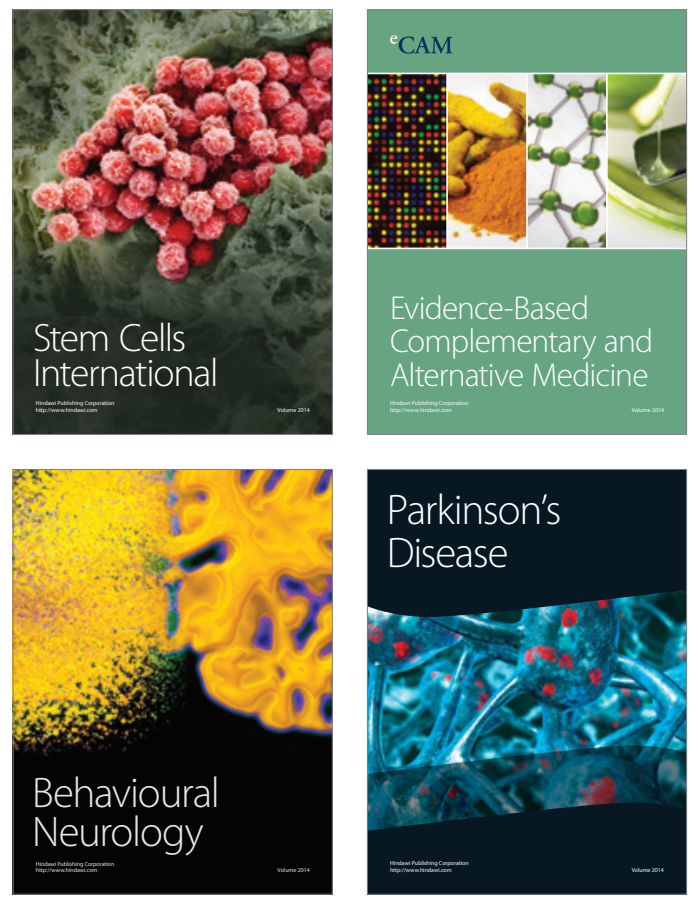
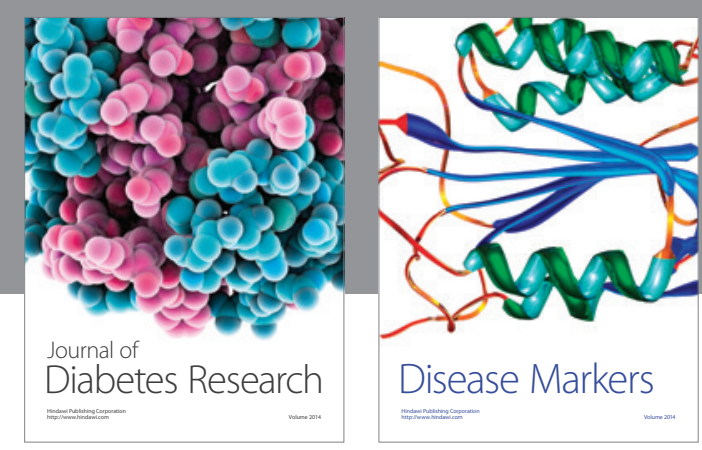

Disease Markers
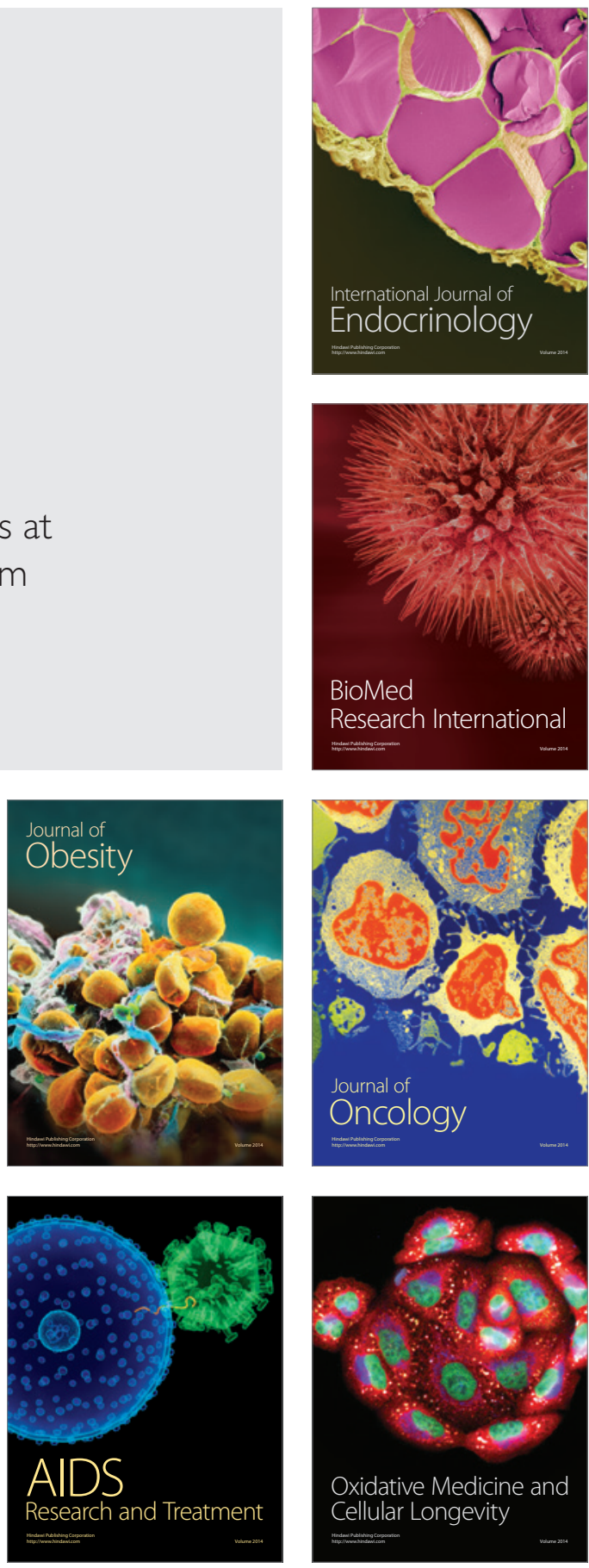